Hasking, Position paper for guiding response to non-suicidal self-injury in schools, 'School Psychology International' $(37,6)$ pp. 644-663. Copyright @ 2016 . DOI: $10.1177 / 0143034316678656$.

\title{
Position paper for guiding response to non-suicidal self-injury in schools
}

\section{Abstract}

Around the world, school staff are increasingly expressing concern about nonsuicidal self-injury (NSSI) and how best to address this behavior in the school setting. However, there is a notable lack of informed guidance for schools, and clear inconsistencies in the practices school staff adopt. In this position paper we draw on our collective research and clinical expertise to provide best-practice guidelines for addressing NSSI in school settings. We outline the importance of a school protocol, and the key features all school protocols should contain. We also focus on how schools can minimize contagion of NSSI within their school environment. We believe these guidelines will be an important starting point for schools interested in developing an evidence-based approach to addressing NSSI.

\section{Keywords}

NSSI, policy, school staff

Nonsuicidal self-injury (NSSI), the deliberate destruction of one's body tissue in the absence of suicidal intent, is a complex behavior frequently met with confusion and misunderstanding (ISSS, 2007). Although often associated with mental illness including anxiety disorders, major depression, eating disorders, and the develop- ment of personality disorders, NSSI also occurs outside the context of psycho- logical illness, and is common among school-based youth (e.g. Lewis \& Heath, 2015). Although NSSI is distinct from suicide, it confers significant risk for suicidal thoughts and behavior (e.g. Whitlock et al., 2013). Thus, NSSI represents a para- mount mental health concern among school-aged youth and merits attention from school mental health professionals. 
Due to its high prevalence in adolescence and its association with deteriorating academic performance, being bullied, and poor social connectedness (Garisch \& Wilson, 2015; Martin, Richardson, Bergen, Roeger, \& Allison, 2005; Rotolone \& Martin, 2012), NSSI merits attention within the school context (Heath, Toste, Sornberger, \& Wagner, 2011). However, school staff express uncertainty and help- lessness when it comes to addressing NSSI with students, and are calling for clear school guidelines (Berger, Hasking, \& Reupert, 2014a; Berger, Hasking \& Reupert, 2014b; Heath, Toste, \& Beettam, 2006).

Therefore, in this paper we draw on the most recent research, existing school protocols, and our combined expertise in working with schools, to provide best- practice guidelines for addressing NSSI in school settings. We begin with a brief overview of the nature and extent of NSSI among secondary school students, including why young people self-injure, consequences of self-injury, and how NSSI relates to suicidal behavior. We then propose a unique role for schools in addressing NSSI, and outline the key elements we believe are essential to school protocols that effectively address NSSI. These include detecting and responding to NSSI, appropriate referral, and guidelines for the involvement of parents/guardians. We also address the potential contagion of NSSI within schools (Miller \& Brock, 2010; Walsh, 2012). Finally, we consider the grounding ethical principles that underlie an effective response to NSSI in schools.

\section{Part 1. The nature and extent of NSSI}

Research on NSSI has historically been hampered by definitional inconsistency, restricting comparison of findings across different studies. Recent inclusion of NSSI in the Diagnostic and Statistical Manual for Mental Disorders (DSM) as a 'condition for 
Hasking, Position paper for guiding response to non-suicidal self-injury in schools, 'School Psychology

International' $(37,6)$ pp. 644-663. Copyright @ 2016 . DOI: $10.1177 / 0143034316678656$.

further study' offers potential to further tighten our understanding of what NSSI involves (DSM-5; APA, 2013). According to the DSM-5 criteria, NSSI is undertaken without suicidal intent and includes behaviors that are not socially sanctioned, thus excluding tattooing and body piercing.

Commensurate with this conceptualization of NSSI, behaviors most often reported include cutting, scratching or burning the skin as well as hitting or bruising oneself (Swannell, Martin, Page, Hasking, \& St John, 2014). NSSI has only recently been identified as a highly prevalent behavior among youth, beginning with the first school study in 2002 (Ross \& Heath, 2002). Approximately $18 \%$ of school-based adolescents report selfinjuring at least once (Muehlenkamp, Claes, Havertape, \& Plener, 2012; Swannell et al., 2014) with up to one-third of these youth reporting repetitive NSSI (Andrews, Martin, Hasking, \& Page, 2013; Ross \& Heath, 2002).

\section{Why do young people self-injure?}

Unfortunately, NSSI is often perceived as attention seeking and manipulative despite ample evidence against such views (for a review see Klonsky, 2007).

Notwithstanding the potential impact of these misconceptions (e.g. stigma; Lewis, Michal, Mahdy, \& Arbuthnott, 2014), a substantial body of evidence indicates that youth engage in NSSI for a multitude of reasons, which broadly fall within intrapersonal or social domains (e.g. Klonsky, 2007; Nock \& Prinstein, 2004).

The most commonly cited reasons for NSSI fall within the intrapersonal domain. In particular, youth who self-injure most often indicate using NSSI as a means to regulate (and obtain relief from) unwanted affective and cognitive experiences (e.g. distress, anxiety, negative self-views) that are perceived to be intolerable (Klonsky, 2007; Lewis 
\& Santor, 2008). The second most commonly reported reason for NSSI is to punish oneself and/or to express hatred toward the self (Klonsky, 2007). Other intrapersonal reasons for NSSI include, but are not limited to, diminishing feelings of depersonalization or dissociation, generation of feelings when experiencing emotional emptiness, and forestalling suicidal ideation, impulses, and urges (Klonsky, 2007; Nock \& Prinstein, 2004).

Ongoing research into the neuro-biological correlates of NSSI, and how these interact with psychological mechanisms, supports the affect-regulatory function of NSSI. Despite some inconsistencies, results from psychophysiological and imaging studies, and exploration of neurotransmitter activity in people who self-injure, generally support the notion that NSSI is primarily used to down-regulate aversive emotions (Groschwitz \& Plener, 2012; Kaess, et al., 2012). In addition, there is robust evidence for alterations in pain perception/tolerance in individuals who self-injure, but insufficient knowledge regarding its exact mechanisms (Koenig, Thayer, \& Kaess, 2016). Overall, although rapidly progressing, this work is in its infancy and more is needed to better understand the complex interplay between the biological and psychological mechanisms underlying NSSI.

Although not as common, social reasons should also be considered when working with youth who self-injure. Social reasons for NSSI often include difficulty with interpersonal problem solving or communication. For instance, youth may self- injure in an effort to communicate their distress to others (Klonsky, 2007; Nock \& Prinstein, 2004); this may occur when previous attempts to communicate distress have failed (Nock, 2008). Consistent with this perspective is research in which youth who self-injure 
Hasking, Position paper for guiding response to non-suicidal self-injury in schools, 'School Psychology International' $(37,6)$ pp. 644-663. Copyright @ 2016 . DOI: $10.1177 / 0143034316678656$.

report more difficulty resolving interpersonal situations than youth who do not self-injure (Nock \& Mendes, 2008). While there are several discrete functions which maintain NSSI, it is important to bear in mind that rea- sons for NSSI may vary over time, and that many youth report more than one NSSI function.

\section{Consequences of NSSI}

The most obvious consequences of NSSI are the injuries incurred, which may result in permanent scarring (Lewis, 2016). Among the psychological consequences of NSSI is the potential worsening of mental health difficulties, including anxiety, depression, and emotion dysregulation (Lewis \& Heath, 2015). Continued NSSI may also exacerbate social difficulties such as isolation (Hasking, Rees, Martin, \& Quigley, 2015). The gravest consequence of NSSI is the risk for suicide.

\section{The relation between NSSI and suicide}

As noted earlier, NSSI is distinct from suicidal behaviour in terms of its underlying reasons wherein the former is not engaged in to end one's life and the latter is. Notwithstanding these motivational differences, a significant number of young people who self-injure also report having attempted suicide in the past (Nock, Joiner, Gordon, Lloyd-Richardson, \& Prinstein, 2006). Indeed, in one study, NSSI was found to confer a seven-fold increase in the risk for attempted suicide (Guan, Fox, \& Prinstein, 2012) and recent research has highlighted NSSI as a unique suicide risk factor (Klonsky, May, \& Glenn, 2013). Among some of the identified NSSI risk factors for suicide are: Using multiple methods of NSSI, having a longer-standing NSSI history, and/or reporting little to no physical pain at the time NSSI occurs (Nock et al., 2006). It is therefore incumbent upon school mental health professionals to assess for suicide risk when working with 
youth who self-injure, both upon initial discovery of NSSI and on an ongoing basis.

\section{Part 2. School response}

Identifying NSSI in young people in schools provides a critical window of opportunity to engage at-risk youth in conversation, link to services, and create on-site care and management plans. Shaffer and Gould (2000) argued that school is the most suitable place for prevention of both suicidal and non- suicidal injury, because (i) such behavior is most common among adolescents;

(ii) most adolescents go to school, which allows access to the majority of at-risk individuals; and (iii) prevention and treatment in schools may be less stigmatizing than interventions conducted in specialized centers for mental healthcare. Further, NSSI and associated difficulties commonly emerge in the school con- text, where teachers and other school professionals often represent the point of first contact (Berger, Reupert, \& Hasking, 2015; Plener, Kaess, Bonenberger, Blaumer, \& Spro“ber, 2011).

In most parts of the world, schools tend to neglect the topic of mental health and, particularly, forms of self-harm including NSSI. Reasons for this are stigma, lack of information, and insecurity about how to handle these behaviors. In the US, Australia, and UK many schools have guidelines and protocols for addressing suicidal thinking and behavior, but known differences in aetiology, frequency, methods, medical severity, and functions of suicidal and non-suicidal self-injury underscore the need for distinct protocols that can be implemented in schools (Andover, Morris, Wren, \& Bruzzese, 2012). Conflating NSSI and suicide proto- cols risks detrimental ends for youth, families, and school personnel. For example, although suicide-focused protocols indicate sending a student at high suicide risk directly to a hospital for emergency intervention, this is rarely 
Hasking, Position paper for guiding response to non-suicidal self-injury in schools, 'School Psychology

International' $(37,6)$ pp. 644-663. Copyright @ 2016. DOI: 10.1177/0143034316678656.

warranted in NSSI and may decrease the likelihood that a student who self-injures seeks assistance when injuries are in need of medical attention.

\section{Key elements of a school response to NSSI}

\section{School protocol}

A protocol provides steps to guide school personnel in responding to students who self-injure. It is important in facilitating timely, appropriate, and consistent response to occurrences of NSSI within the school. There has been increasing attention paid to the development of NSSI school protocols and guidelines over the past decade with certain elements being repeatedly identified as essential (see Berger, Hasking, \& Reupert, 2015 for a review). Recently, in the first empirical evaluation of a NSSI school policy by school staff, Berger and colleagues (2015) reported a generally positive response to the school policy, suggesting that an increasing number of school staff recognize the need for specific NSSI protocols. Drawing on this body of literature and the authors' collective experience with NSSI in schools across multiple contexts we have identified key elements for school protocols (Table 1).

School protocols to address NSSI should clearly outline the roles and responsibilities of all staff and establish a team to coordinate case management for students who self-injure while taking into account school culture, resources and policies. This could involve identifying one or two trained individuals as the 'point persons' to handle all initial issues. This team must be capable of conducting a thorough suicide risk assessment and to make necessary referrals. A school proto- col should also articulate the guidelines regarding when parents/guardians are to be notified, and how parents can be involved in addressing NSSI. While it is often the case that geographic regions, school 
boards, and even individual schools have varying policies with respect to when to notify parents regarding NSSI, it is critical that there be an agreed upon protocol to address this issue. As part of an effective response schools also play a role in providing general information about NSSI to parents. Further, given that research indicates that practitioners with less education about self-injury may feel more judgmental towards, and less able to respond to youth who self-injure (e.g. Heath et al., 2006), we recommend that all school personnel be provided with professional development regarding self-injury as part of an institution-wide practice. Finally, school protocols can outline strategies to minimize contagion of NSSI among students.

\section{Responding effectively}

School mental health professionals commonly believe that the first step to respond to NSSI within the school is to accurately identify or detect students' self-injury. However, identification poses significant challenges if the youth does not self-identify, and students most commonly choose to hide evidence of their self-injury. Consequently, efforts to identify or confirm the behavior's presence needs to be managed with tremendous sensitivity as students may find these efforts intrusive and humiliating. Thus, although it is often recommended that professionals be aware of possible 'signs' of NSSI such as: (a) unexplained cuts, burns, and/or bruising; (b) inappropriate dress for season and/or avoidance of activities that require the removal of clothing (e.g. swimming, gym); (c) school work with a focus on NSSI (e.g. poetry, art, stories), it is important to be aware that there is no single sign of NSSI. In addition, many of these examples will not be obvious until the youth is ready to seek help. 
Hasking, Position paper for guiding response to non-suicidal self-injury in schools, 'School Psychology International' $(37,6)$ pp. 644-663. Copyright @ 2016. DOI: 10.1177/0143034316678656.

Following confirmation of a student's self-injury, initial response by the school professional is critical. Because the response to NSSI disclosure or discovery can shape subsequent student perception and receptiveness to intervention, it is particularly important that school personnel understand how to maintain an empathic, neutral, and supportive demeanour.

First conversations can have many different tones and are often emotionally charged. Validating the behaviour, and the feelings or thoughts underpinning the NSSI, is important. Communicating an understanding that the young person is doing their best and that their self-injury serves a purpose is critical. It is also important to acknowledge and convey that NSSI can be difficult to stop for the youth; therefore, discussions about stopping selfinjury should not occur at this time. Rather, the focus should be on the difficulties underlying the self-injury. Specifically, a respectful curiosity is recommended (Kettlewell, 1999; Walsh, 2012); this involves conveying a genuine interest in wanting to understand what is behind the young person's self-injury (e.g. can you help me understand about your self-injury?). Presuming that a young person will simply 'outgrow' the behavior, that they should 'just stop', or that it can be written off as 'attention seeking' are all unhelpful.

If the young person does not want to talk, it is best to avoid pressuring the youth. NSSI can evoke strong feelings of shame, and defensiveness which, in turn, may result in expressions of anger or withdrawal. It is important to bear in mind that many youth who self-injure have difficulty communicating and discussing their emotional experiences (e.g. Nock, 2008). Because of this, it is essential that the professional working with the young person persist in reaching out to the student and letting the student know that 
he/she is available for support.

If a student's self-injury was disclosed by a fellow student, this student will also need an effective response. Acknowledging the decision to confide in a staff member, perhaps against a friend's wishes, is important and the student will need to know they have done the 'right thing'. This student, and other peers, may also be referred to the selfinjury team or mental health staff to help address their own reactions to their friend's selfinjury.

\section{Appropriate referral}

Knowing who the designated point person is for managing NSSI within the school (as described in the school protocol, Table 1), and how to quickly locate her/him is critical for all school staff. This person, together with school psychologists and other mental health staff, can play a vital role in addressing NSSI among students. For some students, school-based counselling around issues underlying the self- injury will be sufficient. For others, referral to external supports will be warranted. A detailed discussion of psychological treatment for NSSI is beyond the scope of this paper. However, it is worth noting that recent reviews of the treatment literature indicate that, given a lack of well-controlled trials, there is no empirical evi- dence to support any one form of treatment as 'gold-standard', especially among adolescents (Glenn, Franklin, \& Nock, 2015; Turner, Austin, \& Chapman, 2014). Among adults, dialectical behaviour therapy, cognitive behaviour therapy and emotion-regulation group therapy offer some promise as treatment options. Common components of effective treatments include skills training (e.g. in emotion regulation), motivation for treatment, and a focus on improving family and other interpersonal relationships (Glenn et al., 2015; Turner et al., 2014). 
Hasking, Position paper for guiding response to non-suicidal self-injury in schools, 'School Psychology

International' $(37,6)$ pp. 644-663. Copyright @ 2016 . DOI: $10.1177 / 0143034316678656$.

Importantly, there are no randomized controlled trials of treatment approaches conducted in a

school setting, or with school-based youth.

Anecdotally, some clinicians report the use of replacement behaviours (e.g. marking red lines on the skin with a marker, holding ice cubes) to be helpful in treating NSSI. However, at this stage, there is no scientific evidence to support their efficacy, and there is anecdotal evidence that such practices may actually be harmful for some youth. Further, replacement behaviours do not address the underlying issues that initiate or maintain NSSI. As such, until we have evidence regarding their utility, or otherwise, we do not endorse the use of replacement behaviours as a treatment strategy.

Similarly, some clinicians use a 'no self-injury contract' which requires young people to promise not to self-injure under any circumstances. This contract is then usually countersigned by the clinician, student, and parents. As with replacement behaviours there is no evidence 'no self-injury' contracts are effective. These con- tracts can instead promote secrecy and result in a failure to confide future episodes of NSSI. As school students are already reluctant to disclose their NSSI (Hasking et al., 2016), 'no self-injury contracts' could have similar detrimental effects for young people. Rather than use these contracts, we recommend school mental health staff implement safety plans. Safety plans focus on working collaboratively with the young person to identify supports in their environment, identifying triggering situations, rehearsing alternate coping strategies and providing the young person with emergency contacts if required. While 'no self-injury contracts' can appear punitive, the collaborative approach adopted in safety plans is more likely to empower and support the young person, and encourage more healthy coping 
techniques.

Clearly, more work is needed to determine the most efficacious approach to treating NSSI among school-based youth. For the purposes of this paper, we emphasize the need for all school staff to know how to appropriately respond to a student who selfinjures, to refer to the designated point person, and for that person to have clear referral pathways to individuals or services with experience working with youth who self-injure.

\section{Engaging parents/guardians}

Determining the point at which to notify parents/guardians that their child selfinjures may be complicated, and school professionals must carefully weigh the youth's risk profile alongside relevant legal obligations (e.g. to schools, parents/ guardians) and ethical issues (e.g. privacy, confidentiality). This is compounded when considering the various professions involved in working with youth in school settings, who may hold different views, concerns, and, in some cases, need to abide by different ethical and legal codes. As such, whether to notify parents warrants case-by-case consideration.

In most cases, it is in the best interests of the adolescent to notify parents of their child's self-injury, and to engage them in the recovery process. However, while most parents want to know that their child is engaging in NSSI, it is important to bear in mind that, in some cases, breaking confidentiality may yield additional (albeit unintended) harm for the student. For instance, many youth who self-injure feel alienated and may already be distressed about their NSSI being discovered.

In these cases, parent notification may amplify these feelings (White Kress, Costin, \& Drouhard, 2006). Accordingly, mental health professionals working with youth who self-injure in schools are in an often difficult position, as they must consider at what 
point parents and school administrators should be privy to confidential information about the student.

Clearly, in the event that a youth expresses imminent risk for suicide, it is imperative to involve parents/guardians. In these cases, the school mental health professional should be involved in the initial parent/guardian contact (Toste \& Heath, 2010), and immediate professional mental health care must be sought out- side the school environment. However, when a youth is not at high risk for suicide, it may be less clear when (or if) to involve parents/guardians. For example, some suggest that if a student is at low suicide risk (determined following a suicide risk assessment), and does not present with serious mental health symptoms, then NSSI, like other high-risk behaviors (e.g. alcohol use), may not indicate parent/ guardian involvement (Berger et al., 2015; Lieberman, Toste, \& Heath, 2009; Nixon \& Heath, 2009). An important caveat to this, however, is that youth who self-injure but do not present with high risk for suicide often experience significant distress. These youth—like all youth who engage in NSSI—should receive therapeutic support and be followed-up by either school or non-school mental health professionals for assessment. This is essential in terms of ensuring the level of suicide risk for that young person has not changed. With evidence to suggest that the trajectory of NSSI may change over time (Plener, Schumacher, Munz, \& Groschwitz, 2015), it is important not to assume that a student's level of risk will remain static.

When parent/guardian contact is warranted, it is strongly recommended that the student be informed that his/her parent(s)/guardian(s) will be notified and to involve the student in the process. This could include having the student present during the phone call to parents/guardians as it allows students to be aware of the nature of the 
communication and may mitigate feelings of trust being broken or feeling further alienated (Bubrick, Goodman, \& Whitlock, 2010; Nixon \& Heath, 2009). In some cases, it may also mean encouraging the student to share that he/she has self-injured to the parent/guardian with the support and presence of the school staff, or if possible, a mental health professional.

Once parents/guardians become aware that their child is self-injuring, it is incumbent on the school mental health professional to discuss the best ways to provide a supportive environment for the youth. One part of this conversation should involve psycho-education about the types of responses that can be helpful (and unhelpful) when discussing NSSI, with the ultimate goal of helping to foster an empathic and supportive relationship with their youth with respect to NSSI (Walsh, 2012).

Inasmuch as NSSI impacts youth, it also impacts parents (Arbuthnott \& Lewis, 2015; Kelada, Hasking, \& Melvin, 2016). This is often the first time parents/guardians become aware of their child's NSSI. Unsurprisingly, and upon discovery that their youth self-injures, parents often experience myriad reactions, including but not limited to: Anxiety, sadness, shock, anger, distress, guilt, helplessness, and disbelief (Kelada, Whitlock, Hasking, \& Melvin, 2016). Hence, it is important to ensure that parents/guardians receive resources that can help to normalize and understand their reactions, understand why youth self-injure, and discuss how to support their youth while also taking care of themselves (see Resources at end; Arbuthnott \& Lewis, 2015).

\section{Understanding and managing social contagion of NSSI in schools}

While school can certainly be a helpful place for prevention and early detection of NSSI, school is also known to represent a place where 'NSSI contagion' can occur (e.g. 
Lieberman et al., 2009). Walsh and Rosen have defined social contagion of self-injury in two ways: (1) when acts of NSSI occur in two or more persons within the same group within a 24-hour period, and (2) when acts of NSSI occur within a group in statistically significant clusters or bursts (Walsh \& Rosen, 1985). These two definitions have different emphases and are not incompatible. The key point about social contagion of self-injury in schools is that multiple youth self-injure within a short time period.

\section{Identifying youth susceptible to social contagion}

Identifying individuals susceptible to social contagion of NSSI requires returning to the functions of the behavior previously discussed. Logically, youth who present with persistent emotional distress are likely to be drawn to self-injury, to peers who engage in self-injury, and to media sources that focus on NSSI. In some cases, youth who are socially disconnected, isolated, and alienated may be especially drawn to similarly alienated peers (Prinstein et al., 2010).

\section{Strategies for intervening}

School professionals are encouraged to consider three main approaches in order to minimize the risk of contagion:

Reducing communication about self-injury. Reducing communication about NSSI among students is generally recommended (Nixon \& Heath, 2009; Walsh, 2012). However, it is also important to recognize that NSSI is frequently dis- cussed amongst students and within peer groups. Thus, it may be more effective to work towards influencing the nature of the communication. This involves suggesting to students that they reduce communication that focuses primarily on the details of the self-injury (e.g. particular methods, what happens during an NSSI episode). Alternatively, it can be 
helpful for school professionals to encourage students to focus on seeking support from their peers about the feelings that may underlie NSSI, without direct focus on the NSSI itself. Alongside this, it can be useful to explain to students that there is evidence that in certain instances sharing details (e.g. in conversation, texting, online) about NSSI can be triggering to vulnerable students. That is, some young people may experience NSSI urges upon being exposed to details about NSSI (Baker \& Lewis, 2013; Lewis \& Knoll, 2015). Responding to students' showing scars or wounds. Schools often have significant concern about students who elect to not conceal their NSSI scars or wounds. In particular, there is concern that viewing NSSI scars or wounds can be triggering for vulnerable students. Although this is a very new area of research, there is at least some evidence that among those who engage in NSSI viewing scars may not be triggering. In contrast, viewing fresh wounds carries a stronger likelihood of being triggering (Baker \& Lewis, 2013). Furthermore, there is growing evidence that the accepting and choosing to stop concealing one's scars can be very therapeutic and an important step in recovery (Lewis, 2016; Lewis \& Mehrabkhani, 2016). In light of the complexity of the current research in this area, it is recommended that students be asked to cover wounds, due to school health requirements concerning any potential contamination by blood which applies to all cuts or wounds; secondly it should be explained to students that there is some evidence that the viewing of wounds by those who may be still struggling with their recovery around self-injury could be triggering. However, response to scaring necessitates a different approach, with recognition that a student's choice to stop concealing their scars may be a very positive step in their recovery, as concealment of scars may be associated with the shame of having self-injured (Lewis \& Mehrabkhani, 2016). Although this may be the 
case, dis- playing one's scars may have unintended consequences, such as intrusive questions, negative comments, or even bullying. Therefore, it is imperative that school mental health professionals have a sensitive and compassionate discus- sion with the student. This involves: a) acknowledging that this is the student's choice and that this may be a positive step for him/her and b) reviewing the potential negative consequences that may ensue, and discussing how these potential challenges could be addressed and how the student can be supported. In the event that the student chooses to no longer conceal their scars, it can be detrimental to the student's well-being to insist that scars are hidden.

Treating self-injury using individualized methods, not groups. While there is concern about increasing risk of NSSI when discussed in a group format, school-based prevention programs, when run well, do not produce iatrogenic effects (Jacobs, Walsh, \& Pigeon, 2009; Muehlenkamp, Walsh, \& McDade, 2010). Yet, group treatment of NSSI in students who already self-injure can be risky because open discussion of the antecedents, behavior, and consequences of the behavior runs the risk of triggering other group members. A more strategic course is to refer students to individual therapy where they can focus on identifying the antecedents for their NSSI and acquire emotion regulation and interpersonal effectiveness skills. A notable exception is group work for replacement skills training (e.g. Dialectical Behavior Therapy), where the focus is on learning, practicing, and generalizing skills in the real world (Rathus \& Miller, 2015; Walsh, 2012).

\section{Final considerations}

\section{Ethical Issues}


Self-injury is a topic of considerable sensitivity for teachers, pastoral care workers, parents and family, health and educational services, and others, not least of all people who hurt themselves and their confidantes. Given concerns over the possibilities of contagion as noted above, and the nature and extent of risk that might be associated with NSSI, all of us have encountered uncertainty and concern over appropriate and ethical care of young people who self-injure. Issues include privacy and the propriety of involving parents upon discovery of self-injury, risk-assessment and management, appropriate treatment (and who should deliver it), and whether NSSI is a topic for broader discussion in schools. These issues are not dissimilar from those associated with conducting research on self-injury, which are dealt with in greater depth elsewhere (Lloyd-Richardson, Lewis, Whitlock, Rodham, \& Schatten, 2015). Generally speaking, people whose practice focuses on student well-being belong to occupations that adhere to codes of ethics that tend to share common principles. In practice, this means ensuring that young people have the right to help and to decide to access that help, to privacy and to safety, subject to concerns around risk, and it is this last that generates the most concern. We would argue that it is important to see youth as collaborators in the intervention process and that giving choices about potential next steps, and ensuring respectful language and demeanor, are not only good ways to ensure ethical, but also effective, practice (Walsh, 2012). Involvement of young people in the development, and piloting, of a school response to NSSI is consistent with ethical practice, and assists in giving a sense of involvement in decision-making, rather than as the subjects of others' decisionmaking.

\section{Conclusion}


Despite increasing concern among school staff regarding NSSI among students, there are few clear guidelines to assist staff. In this position paper we have endeavoured to gather the available evidence to suggest guidelines for how best to address NSSI in school contexts, that we hope schools will use as a framework for their own discussions about how to manage NSSI in their own schools. While we have attempted to tackle the most pressing issues facing schools today, it is important to acknowledge that no one set of guidelines will meet the needs of all schools. Local legislation, education department policies and cultural considerations will necessarily drive implementation of these guidelines. At the very least, we suggest discussion concerning the development of an explicit and clearly-communicated school protocol, training for all staff regarding NSSI, and consideration of the complexities concerning efforts to minimize contagion of NSSI in the school con- text is essential for all schools wishing to respond effectively to NSSI. 


\section{References}

American Psychiatric Association. (2013). Diagnostic and Statistical Manual of Mental Disorders. Fifth Edition. Arlington, VA: American Psychiatric Association.

Andover, M. S., Morris, B. W., Wren, A., \& Bruzzese, M. E. (2012). The co-occurrence of nonsuicidal self-injury and attempted suicide among adolescents: Distinguishing risk factors and psychosocial correlates. Child and Adolescent Psychiatry and Mental Health, 6, 11. doi: 10.1186/1753-2000-6-11.

Andrews, T., Martin, G., Hasking, P., \& Page, A. (2013). Predictors of continuation and cessation of non-suicidal self-injury. Journal of Adolescent Health, 53, 40-46. doi: 10.1016/j.jadohealth.2013.01.009.

Arbuthnott, A. E., \& Lewis, S. P. (2015). Parents of youth who self-injure: A review of the literature and implications for mental health professionals. Child and Adolescent Psychiatry and Mental Health, 9, 35. doi:10.1186/s13034-015-0066-3.

Baker, T. G., \& Lewis, S. P. (2013). Responses to online photographs of non-suicidal selfinjury: A thematic analysis. Archives of Suicide Research, 17, 223-235. doi: 10.1080/ 13811118.2013.805642.

Berger, E., Hasking, P., \& Reupert, A. (2014a). 'We're working in the dark here': Knowledge, 
Hasking, Position paper for guiding response to non-suicidal self-injury in schools, 'School Psychology

International' $(37,6)$ pp. 644-663. Copyright @ 2016. DOI: 10.1177/0143034316678656.

attitudes and response of school staff towards adolescents' self-injury. School Mental Health, 6, 201-212. doi: 10.1007/s12310-013-9114-4.

Berger, E., Hasking, P., \& Reupert, P. (2014b). Response and training needs of school staff towards students' self-injury. Teaching and Teacher Education, 44, 25-34. doi: 10.1016/ j.tate.2014.07.013.

Berger, E., Hasking, P., \& Reupert, A. (2015). Developing a policy to address non-suicidal selfinjury in schools. Journal of School Health, 85, 629-647.

Berger, E., Reupert, A., \& Hasking, P. (2015). Pre-service and in-service teachers' know- ledge, attitudes, and confidence towards self-injury among students. Journal of Education for Teaching, 41, 37-51. Available at http://dx.doi.org/10.1080/02607476.2014.992633.

Bubrick, K., Goodman, J., \& Whitlock, J. (2010). Non-suicidal self-injury in schools:

Developing and implementing school protocol. Cornell Research Program on SelfInjurious Behavior in Adolescents and Young Adults. Available at http://www. selfinjury.bctr.cornell.edu/documents/schools.pdf.

Garisch, J. A., \& Wilson, M. S. (2015). Prevalence, correlates, and prospective predictors of nonsuicidal self-injury among New Zealand adolescents: Cross-sectional and longitu- dinal survey data. Child and Adolescent Psychiatry and Mental Health, 9, 28. doi: 10.1186/ s13034-015-0055-6.

Glenn, C. R., Franklin, J. C., \& Nock, M. K. (2015). Evidence-based psychosocial treat- ments for self-injurious thoughts and behaviors in youth. Journal of Clinical Child and Adolescent Psychology, 44, 1-29. doi: 10.1080/15374416.2014.945211.

Groschwitz, R. C., \& Plener, P. L. (2012). The neurobiology of non-suicidal self-injury (NSSI): A review. Suicidology Online, 3, 24-32. 
Guan, K., Fox, K. R., \& Prinstein, M. J. (2012). Nonsuicidal self-injury as a time-invariant predictor of adolescent suicide ideation and attempts in a diverse community sample.

Journal of Consulting and Clinical Psychology, 80, 842-849. doi: 10.1037/a0029429.

Hasking, P., Rees, C., Martin, G., \& Quigley (2015). What happens when you tell someone you self-injure? The effects of disclosing NSSI to adults and peers. BMC Public Health, 15, 1039. doi: 10.1186/s12889-015-2383-0.

Heath, N. L., Toste, J. R., \& Beettam, E. L. (2006). 'I am not well-equipped”': High school teachers' perceptions of self-injury. Canadian Journal of School Psychology, 21, 73-92. doi: $10.1177 / 0829573506298471$.

Heath, N. L., Toste, J. R., Sornberger, M. J., \& Wagner, C. (2011). Teachers' perceptions of nonsuicidal self-injury in the schools. School Mental Health, 3, 35-43. doi: 10.1007/ s12310010-9043-4.

International Society for the Study of Self-Injury. (ISSS; 2007). Definition of non-suicidal selfinjury. http://www.itriples.org/isss-aboutself-i.html

Jacobs, D. G., Walsh, B. W., \& Pigeon, S. (2009). Signs of self-injury prevention program. Wellesley, MA: Screening for Mental Health and The Bridge. Retrieved from http:// www.mentalhealthscreening.org/programs/youth-prevention-programs/sosi/

Kaess, M., Hille, M., Parzer, P., Maser-Gluth, C., Resch, F., Brunner, R. (2012). Alternations in the neuroendocrinological stress response to acute psychosocial stress in adolescents engaging in nonsuicidal self-injury. Psychoneuroendocrinology, 37, 157-161. doi: 10.1016/j.psyneuen.2011.05.009.

Kelada, L., Hasking, P., \& Melvin, G. (2016). The relationship between nonsuicidal self- injury and family functioning: Adolescent and parent perspectives. Journal of Marital and 
Hasking, Position paper for guiding response to non-suicidal self-injury in schools, 'School Psychology

International' $(37,6)$ pp. 644-663. Copyright @ 2016 . DOI: $10.1177 / 0143034316678656$.

Family Therapy, 42, 536-549. doi: 10.1111/jmft.12150.

Kelada, L., Whitlock, J., Hasking, P., \& Melvin, G. (2016). Parents' experiences of non- suicidal self-injury among adolescents and young adults. Journal of Child and Family Studies, 25, 3403-3416. doi: 10.1007/s10826-016-0496-4.

Kettlewell, C. (1999). Skin game: A memoir. New York, NY: St Martin’s Press.

Klonsky, E. D. (2007). The functions of deliberate self-injury: A review of the evidence.

Clinical Psychology Review, 27, 226-239. doi: 10.1016/j.cpr.2006.08.002.

Klonsky, E. D., May, A. M., \& Glenn, C. R. (2013). The relationship between nonsuicidal selfinjury and attempted suicide: Converging evidence from four samples. Journal of Abnormal Psychology, 122, 231-237. doi: 10.1037/a0030278.

Koenig, J., Thayer, J. F., \& Kaess, M. (2016). A meta-analysis on pain sensitivity in self- injury. Psychological Medicine, 46, 1597-1612. doi: 10.1017/S0033291716000301.

Lewis, S. P. (2016). The overlooked role of self-injury scars: A commentary and suggestions for clinical practice. Journal of Nervous and Mental Disease, 204, 33-35. doi: 10.1097/ NMD.0000000000000436.

Lewis, S. P., \& Heath, N. L. (2015). Non-suicidal self-injury among youth. Journal of Pediatrics, 3, 526-530. doi: 10.1016/j.jpeds.2014.11.062.

Lewis, S. P., \& Knoll, A. (2015). Do it yourself: Examination of self-injury first aid tips on YouTube. Cyberpsychology, Behavior, and Social Networking, 18, 301-304. doi:10.1089/ cyber.2014.0407.

Lewis, S. P., \& Mehrabkhani, S. (2016). Every scar tells a story: Insight into people's self- injury scar experiences. Counselling Psychology Quarterly, 29, 296-310. Available at: http://dx.doi.org/10.1080/09515070.2015.1088431. 
Lewis, S. P., Michal, N. J., Mahdy, J., \& Arbuthnott, A. E. (2014). Googling self-injury: The state of health information obtained through online searches for self-injury. JAMA Pediatrics, 168(5), 443-449. doi:10.1001/jamapediatrics.2014.187.

Lewis, S. P., \& Santor, D. A. (2008). Development and validation of the Self-Harm Reasons Questionnaire. Suicide and Life-Threatening Behavior, 38, 104-115. doi: 10.1521/ suli.2008.38.1.104.

Lieberman, R. A., Toste, J. R., \& Heath, N. L. (2009). Non-suicidal self-injury in the schools: Prevention and intervention. In M. K. Nixon, \& N. L. Heath (Eds.), Self- injury in youth: The essential guide to assessment and intervention (pp. 195-213). New York, NY: Routledge.

Lloyd-Richardson, E. E., Lewis, S. P., Whitlock, J. L., Rodham, K., \& Schatten, H. T. (2015). Research with adolescents who engage in non-suicidal self-injury: Ethical considerations and challenges. Child and Adolescent Psychiatry and Mental Health, 9, 1-14. doi: 10.1186/s13034-015-0071-6.

Martin, G., Richardson, A. S., Bergen, H. A., Roeger, L., \& Allison, S. (2005). Perceived academic performance, self-esteem and locus of control as indicators of need for assessment of adolescent suicide risk: Implications for teachers. Journal of Adolescence, 28, 75-87. doi: 10.1016/j.adolescence.2004.04.005.

Miller, D. N., \& Brock, S. E. (2010). Identifying, assessing and treating self-injury at school. Springer: New York.

Muehlenkamp, J., Walsh, B., \& McDade, M. (2010). Preventing non-suicidal self-injury in adolescents: The signs of self-injury program. Journal of Youth and Adolescence, 39, 306- 314. doi: 10.1007/s10964-009-9450-8. 
Hasking, Position paper for guiding response to non-suicidal self-injury in schools, 'School Psychology International' $(37,6)$ pp. 644-663. Copyright @ 2016 . DOI: 10.1177/0143034316678656.

Muehlenkamp, J. J., Claes, L., Havertape, L., \& Plener, P. (2012). International prevalence of adolescent nonsuicidal self-injury and deliberate self-harm. Child and Adolescent Psychiatry and Mental Health, 6, 10. doi: 10.1186/1753-2000-6-10.

Nixon, M. K., \& Heath, N. L. (2009). Self-injury in youth: The essential guide to assessment and intervention. New York, NY: Routledge Press.

Nock, M. K., Joiner, T. E., Gordon, K. H., Lloyd-Richardson, E. E., \& Prinstein, M. J. (2006). Non-suicidal self-injury among adolescents: Diagnostic correlates and relation to suicide attempts. Psychiatry Research, 144, 65-72. doi: 10.1016/j.psychres.2006.05.010. Nock, M. (2008). Actions speak louder than words: An elaborated theoretical model of the social functions of self-injury and other harmful behaviors. Applied and Preventive

Psychology, 12, 159-168. doi: 10.1016/j.appsy.2008.05.002.

Nock, M. K., \& Mendes, W. B. (2008). Physiological arousal, distress tolerance, and social problem-solving deficits among adolescent self-injurers. Journal of Consulting and Clinical Psychology, 76, 28. doi: 10.1037/0022-006X.76.1.28.

Nock, M., \& Prinstein, M. (2004). A functional approach to the assessment of self-mutilative behavior. Journal of Counseling and Clinical Psychology, 72, 885-890. doi: 10.1037/0022- 006X.72.5.885.

Plener, P. L., Kaess, M., Bonenberger, M., Blaumer, D., \& Spro“ber, N. (2011). Umgang mit nicht-suizidalem selbstverletzendem Verhalten (NSSV) im schulischen Kontext. Kindheit und Entwicklung, 21, 16-22. doi: 10.1026/0942-5403/a000066.

Plener, P. L., Schumacher, T. S., Munz, L. M., \& Groschwitz, R. C. (2015). The longitudinal course of non-suicidal self-injury and deliberate self-harm: A systematic review of the literature. Borderline Personaltiy Disorder and Emotion Dysregulation, 2, 2. doi: 10.1186/ 
s40479-014-0024-3.

Prinstein, M., Heilbron, N., Guerry, J., Franklin, J. C., Rancourt, D., Simon, V., ... Spirito, A. (2010). Peer influence and nonsuicidal self-injury: Longitudinal results in community and clinically-referred adolescent samples. Journal of Abnormal Child Psychology, 38, 669682. doi: 10.1007/s10802-010-9423-0.

Rathus, J. H., \& Miller, A. L. (2015). DBT skills manual for adolescents. New York, NY: Guilford.

Ross, S., \& Heath, N. (2002). A study of the frequency of self-mutilation in a community sample of adolescents. Journal of Youth and Adolescence, 31, 67-77.

Rotolone, C., \& Martin, G. (2012). Giving up self-injury: A comparison of everyday social and personal resources in past versus current self-injurers. Archives of Suicide Research, 16, 147-158. doi: 10.1080/13811118.2012.667333.

Shaffer, D., \& Gould, M. (2000). Suicide prevention in schools. The international handbook of suicide and attempted suicide. Chichester: Wiley.

Swannell, S., Martin, G., Page, A., Hasking, P., \& St John, N. (2014). Prevalence of non-suicidal self-injury (NSSI) in non-clinical samples; systematic review, meta-analysis and metaregres- sion. Suicide and Life-Threatening Behavior, 44, 273-303. doi: 10.1111/sltb.12070.

Toste, J. R., \& Heath, N. L. (2010). School response to non-suicidal self-injury. The Prevention Researcher, 17, 14-17.

Turner, B. J., Austin, S. B., \& Chapman, A. L. (2014). Treating nonsuicidal self-injury: A systematic review of psychological and pharmacological interventions. Canadian Journal of Psychiatry, 59, 576-585. 
Walsh, B. W. (2012). Treating self-injury: A practical guide 2nd ed. New York, NY: The Guilford Press.

Walsh, B., \& Rosen, P. (1985). Self-mutilation and contagion: An empirical test. American Journal of Psychiatry, 142, 119-120.

White Kress, V. E., Costin, A., \& Drouhard, N. (2006). Students who self-injure: School counsellor ethical and legal considerations. Professional School Counselling, 10, 203209. doi: http://dx.doi.org/10.5330/prsc.10.2.x238g1581p74236q.

Whitlock, J., Muehlenkamp, J., Eckenrode, J., Purington, A., Abrams, G. B., Barreira, P., ... Kress, V. (2013). Nonsuicidal self-injury as a gateway to suicide in young adults. Journal of Adolescent Health, 52, 486-492. Available at: http://dx.doi.org/10.1016/j.jadohealth.2012.09.010. 
Table 1. Key elements in school protocol.

Roles and responsibilities

Outline the roles and responsibilities of all staff in the school for detecting and responding to NSSI.

Establishing a point person and/or team (self-injury team, SIT) with training in NSSI to coordinate all aspects of the case management for students who disclose NSSI, including school wide staff education about NSSI.

The SIT communicates to all school staff the need to refer to the SITwhen staff member has reason to believe a student is engaging in NSSI. The importance of referring directly to the SIT and maintaining confidentiality otherwise is emphasized.

The SIT is responsible for providing follow up, within the constraints of confidentiality, to the first responder staff member following a referral to the SIT informing them that the student was provided with appropriate follow up.

The SIT should be available to staff following an interaction that resulted in the staff having intense feelings or reactions. Referral for the staff as needed should be available.

Risk assessment

The appropriate SIT member conducts an initial risk assessment, to identify possible suicide risk. Based on risk assessment the SIT must determine the next step. At one end of the con- tinuum, with high risk students who may be an imminent risk to themselves, an immediate referral to hospital may be needed. On the other end of the continuum, for a low risk student who may have a few incidents of superficial self-injury, the student could be provided with follow up within the school to explore healthier alternative coping strategies and monitor possible changes in the behavior.

\section{Referral}

The SIT should make the appropriate referral as needed based on the risk assessment and with the involvement of the parent/guardian where appropriate.

The SIT should develop and maintain a list of potential referral options for different common risk profiles, and socio-economic levels.

Parent/guardian notification/involvement Legal regulations regarding student confidentiality/parent notification vary substantially from 
place to place, therefore it is imperative that the school protocol recommendations around parent notification be developed with explicit reference to local regulations.

However, wherever possible it is beneficial if the SIT is able to work with the student to involve parent/guardians as they can be invaluable in providing ongoing support.

When parent/guardians are involved the SIT should be sure to share information and resources about NSSI with them and provide early support.

Managing social contagion

School communication about self-injury should be handled with care and focus on the larger context of unhealthy coping behaviors, with an emphasis on enhancing healthy coping. Talks or materials focusing exclusively on self-injury should be avoided

Peer communication about self-injury should be guided but not banned, being explicitly clear that it is out of concern for others who may be triggered by explicit detail.

Students should be asked to cover wounds wherever possible, again with the explicit emphasis on the need to support others who may be struggling with recovery and the potential for triggering them. 